\title{
Gut Microbiota and Hepatocellular Carcinoma
}

\author{
Xuemei Tao Ning Wang Wenxin Qin \\ State Key Laboratory of Oncogenes and Related Genes, Shanghai Cancer Institute, \\ Renji Hospital, Shanghai Jiao Tong University School of Medicine, Shanghai, China
}

\section{Key Words}

Endotoxemia · Gut-liver axis · Gut microbiota · Hepatocellular carcinoma · Probiotics

\begin{abstract}
Background: Hepatocellular carcinoma (HCC) is a common complication of liver diseases such as those related to viral hepatitis and liver cirrhosis. The gut-liver axis is gaining increasing attention as a key pathophysiological mechanism responsible for the progression of HCC. Here, we will review the data from the published literature to address the association between HCC and gut microbiota. Summary: The presence of high levels of endotoxemia in the blood results in portal hypertension and ensuing hepatocyte damage, thus leading to the development of HCC. Probiotics can be used to treat or prevent the progression of HCC, because they may decrease the counts of gut microbiota and thus improve the endotoxemia. Key Message: Increased bacterial translocation can result in endotoxemia, which may play a critical role in the progression of HCC. Modulation of the gut microbiota by probiotics may represent a new avenue for therapeutic intervention in HCC. Practical Implications: Breakdown in intestinal barrier function and bacterial overgrowth are main events in the development of HCC. When the intestinal barrier function is disrupted, large amounts of bacterial products can enter the liver and induce inflammation through their receptors, leading to liver diseases. Altering the gut microflora has been proposed as an adjunctive therapy to reduce bacterial translocation and prevent progression of HCC. The purpose of this review is to discuss the relationship between gut microbiota and HCC in both pathogenesis and treatment by probiotics.
\end{abstract}




\section{Introduction}

Hepatocellular carcinoma (HCC) is the third leading cause of cancer mortality worldwide $[1,2]$. Major risk factors for HCC include infection with hepatitis B virus (HBV) or hepatitis C virus (HCV); these risk factors lead to formation and progression of cirrhosis, which is present in $80-90 \%$ of HCC patients. The 5-year cumulative risk for the development of HCC in patients with cirrhosis ranges between 5 and 30\% [2]. The gut and liver are key organs in nutrient absorption and metabolism. The liver has a double blood supply, including the hepatic artery which comes from the celiac axis and the portal vein which brings venous blood from the intestines and spleen, while the portal venous blood flow comes from the mesenterium and accounts for $75 \%$ of the liver blood supply [3]. The portal vein delivers gut-derived products, such as lipopolysaccharide (LPS), bacterial DNA and peptidoglycan, to the liver. Because of its anatomical location, the liver is exposed to gut-derived bacterial components.

A number of studies have been dedicated to the mechanism of the gut-liver axis. The gastrointestinal tract is a metabolic and immunological system. It harbors the most complex human microbial ecosystem and is a vast pool of bacteria and endotoxins in the body [4]. The human intestine is believed to contain approximately 100 trillion intestinal microbiota, comprising about 500-1,000 different species [5]. It has been estimated that the microbes in the mucosal surface of the human gastrointestinal tract are colonized by $10^{13-14}$ bacteria with 400 different species and subspecies [6]. The normal gut barrier is made up of the following parts: (1) the normal composition and distribution of intestinal flora, (2) the intestinal mucosal barrier and (3) the integrity of the immune system. Any damage to the gut barrier could increase the intestinal permeability, followed by bacteria translocation and accumulation of LPS [7], which will lead to the pathogenesis of liver diseases. The plasma LPS level was associated with small intestinal bacterial overgrowth, changes in composition of the microbiota and increase in intestinal permeability [8]. Thus, the gastrointestinal tract, functioning as an effective barrier against endotoxin and gut bacteria, may protect the body [9]. Furthermore, the liver also plays an important physiological role in LPS detoxification and, in particular, hepatocytes are involved in the clearance of endotoxin of intestinal derivation [10]. The degradation of endotoxin will be affected if the liver is damaged, and the increase in endotoxin will in turn aggravate liver damage.

Increasing evidence indicates that the gut-liver axis is involved in HCC. Endotoxemia produced by gut microbiota may contribute to hepatocarcinogenesis and may serve as a target to the prevention or treatment of HCC [11]. Herein, we will review the association of gut microbiota with the pathogenesis of HCC.

\section{Roles of Gut Microbiota in Hepatocarcinogenesis}

\section{Viral Hepatitis}

Viral hepatitis, one of the common causes leading to HCC, is the prevailing cause in most parts of Asia and sub-Saharan Africa, especially in China [12]. There is growing evidence that HBV and HCV can accelerate the development of cirrhosis, ultimately leading to HCC. Gut microbiota may contribute to the progression of viral hepatitis and its related complications. Sandler et al. [13] showed in a retrospective study that the degree of liver disease in patients with chronic HBV or HCV infection was associated with microbial translocation.

Increased intestinal permeability, bacterial overgrowth or impaired clearance of microbial products by Kupffer cells may increase the translocation of gut microbiota. Microbiota and their components are able to pass through the gut barrier and enter the portal circulation, and then they are transported to the liver. In the liver, the gut microbial composition can be 
envisaged to result in increased activation of liver Toll-like receptors (TLRs). TLRs are a class of proteins that recognize structurally conserved molecules derived from microbiota [14]. TLR4 is the receptor for LPS. Within the liver, it is well known that Kupffer cells, hepatic stellate cells (HSCs) and hepatocytes express TLR4 and thus might be a target for bacterial LPS, which is an important action on the development and progression of hepatitis [15]. In a mouse model of viral-induced liver disease, germ-free mice were protected from disease and conventional mice were also protected by an antibiotic or the TLR4 antagonist polymixin B [16]. Thus, it seems that the mechanisms by which HBV and perhaps HCV promote liver disease are mediated, in part, via the gut microbiota. Each increased risk for disease progression by LPS-induced inflammation is associated with end-stage liver disease such as HCC in patients with HBV or HCV infection.

\section{Cirrhosis}

The gut is the major source of endogenous bacteria causing infections in advanced cirrhosis [17]. The study of Cirera et al. [18] investigated the risk factors in experimental cirrhosis and explored the mechanism involved in the pathogenesis of patients with bacterial infections. The results showed that translocation of gut organisms to mesenteric lymph nodes was increased in patients with advanced cirrhosis and was reduced to the level found in noncirrhotic patients by selective intestinal decontamination. There was also evidence showing that gut microbiota might promote liver fibrosis in mice, and the increase in bacterial translocation from gut to liver was associated with chemically induced fibrosis [19].

Bacterial translocation takes place in the setting of advanced liver diseases and contributes to the progression of both cirrhosis and its complications [20]. At this stage, the patient has in increased risk of a variety of spontaneous infections, such as spontaneous bacteremia and spontaneous bacterial peritonitis in cirrhosis. These infections are poorly tolerated, since they will accelerate the hyperdynamic circulatory state, increase portal blood pressure and lead to liver failure. Bacterial translocation could also precipitate liver-related complications, such as hepatic encephalopathy. The pathogenesis of hepatic encephalopathy is linked to alterations in gut microbiota and their by-products such as ammonia, indoles, oxindoles, endotoxins, etc. [21, 22]. On the other hand, cirrhosis itself can affect the intestinal barrier and in turn contribute to its own complications [23].

Translocation of bacterial products, such as LPS or bacterial DNA, could stimulate the immune system in cirrhosis patients [24]. LPS levels are commonly increased in patients with liver cirrhosis, and LPS will successively precipitate a cascade, including repeated hepatocytic impairment, hepatic fibrosis, cirrhosis and HCC. There is evidence showing that the malignant degree of liver dysfunction was associated with the level of LPS and bacterial substances [25]. The presence of high levels of LPS and bacterial substances in blood results in hepatocyte damage, ensuring the progression of liver fibrosis [7]. Furthermore, treatment with antibiotics may delay the development of cirrhosis [26]. The activated HSCs express TLR4 and are highly responsive to even low concentrations of LPS [27]. TLR4 will provide a potential cellular link between intestinal derived LPS and liver fibrosis, thus promoting hepatic fibrosis and leading to the development of cirrhosis [28].

HCC

As mentioned above, chronic viral infection or liver cirrhosis remain the major causes of HCC. Gut microbe pathways may contribute to the development of HCC as has been previously demonstrated [29]. However, there is no direct evidence of their role in HCC. Therefore, further studies on a possible link between intestinal microbiota and HCC in humans are needed. 
Bacteria located within the intestinal lumen undergo constant surveillance by TLRs. TLRs are expressed in Kupffer cells, HSCs and hepatocytes and activate these cells to contribute to acute and chronic liver diseases [30]. TLRs are differentially activated by commensal bacteria $[31,32]$, and TLRs will induce pathways involved in gut homeostasis, regulation of tight junctions and antimicrobial peptide secretion [33]. Translocation of bacterial components termed pathogen-associated molecular patterns (PAMPs) triggers inflammatory responses through TLRs in both early and late disease stages [34]. These pattern recognition receptors identify and interact with microbial-associated molecular patterns (MAMPs) on both commensal and pathogenic bacteria. The various MAMPs, including LPS, peptidoglycan and flagellin, are TLRspecific ligands [35], all of which will reach the liver via the portal vein. Notably, hepatic exposure to cancer-promoting MAMPs and metabolites is increased in liver disease and has been linked to intestinal dysbiosis. Accordingly, germ-free status and non-absorbable antibiotics reduce hepatic inflammation, fibrosis and HCC development in mice, whereas treatment with the TLR4 agonist LPS can increase HCC development.

Obesity and high-fat diet have been identified as major risk factors for HCC. Early studies suggested that altered gut microbiota might contribute to obesity by affecting energy harvest from the diet and energy storage in the host [14]. Gut-derived bacterial products are delivered to the liver, where they will activate TLRs and promote a senescence-associated secretory phenotype (SASP) in HSCs, then in turn facilitate the development of HCC [36]. Yoshimoto et al. [37] treated mice with a chemical carcinogen at the neonatal stage to induce a tumor model and demonstrated that gut microbiota were associated with obesity. Administration of antibiotics or gut sterilization could lead to a significant decrease in HCC development in an obesity-related model of hepatocarcinogenesis. Dietary or genetic obesity induced alterations of gut microbiota, thereby increasing the levels of deoxycholic acid (DCA), a gut bacterial metabolite known to cause DNA damage through reactive oxygen species production [38]. The enterohepatic circulation of DCA provoked SASP phenotype in HSCs, which in turn led to the production of proinflammatory cytokines and tumor-promoting factors in the liver that promote HCC, whereas reduction of DCA-producing bacteria by antibiotics decreased the development of HCC [35]. This indicated that the DCA-SASP axis in HSCs facilitated obesityassociated HCC development. Importantly, blocking the production of DCA or depleting the gut microbiota reduced the development of HCC [36].

The role of gut microbiota and TLRs in hepatocarcinogenesis has recently been firmly established by extensive studies. Yu et al. [39] observed an increase in endotoxin levels in experimental HCC models upon administration of the chemical carcinogen diethylnitrosamine (DEN); they found that endotoxin accumulation regulated the survival and proliferation of hepatocytes. Thus, enhanced intestinal permeability to endotoxins appears to be the primary cause of chemically induced endotoxemia. This finding was supported by the fact that reduction of LPS using antibiotics in rats or genetic ablation of its receptor TLR4 in mice dramatically mitigated enteric dysbiosis, decreased liver tumor growth and prevented tumor multiplicity [19]. Yu et al. [39] suggested that reducing gut injury, improving blood flow to the gastrointestinal tract and lessening the gut translocation of endotoxin might improve liver function. Dapito et al. [40] showed that the gut microbiota and LPS/TLR4 pathway play a role in HCC promotion in chronically injured liver. They subjected $\mathrm{C} 3 \mathrm{H} / \mathrm{HeJ}$ and $\mathrm{C} 3 \mathrm{H} / \mathrm{HeOuJ}$ mice to a combination of DEN and the hepatotoxin carbon tetrachloride $\left(\mathrm{CCl}_{4}\right)$, a model that features several characteristics of the cirrhotic environment of chronically injured liver in which human HCC mostly arises [27]. They showed that the intestinal microbiota and TLR4 link inflammation and carcinogenesis in the chronically injured liver. Continuous administration of low doses of LPS increased tumor number and size in conventional wild-type mice, while in gut-sterilized mice systemic LPS levels were reduced and there was less overgrowth of tumor. However, there was no significant contribution of gut microbiota and TLR4 to tumor initiation [41]. 
Patients with liver diseases also have an impact on gut homeostasis, which will lead to gut dysbiosis and gut barrier permeability. Interestingly, Zhang et al. [19] demonstrated that patients with liver cirrhosis and HCC had significantly increased serum endotoxin levels. In the DEN model of rat hepatocarcinogenesis, chronic liver injury was accompanied by imbalance of the gut microflora and tissue damage in intestinal epithelia. Administration of probiotics dramatically mitigated enteric dysbiosis, ameliorated intestinal inflammation and, most importantly, decreased liver tumor growth. In the above-mentioned study of Dapito et al. [40], they suggested that antibiotic-induced gut sterilization could prevent HCC in patients with chronic liver injury. Such a preventive management of cirrhotic patients would require lifelong administration of antibiotics. In fact, approaches based on gut microbiota manipulation have already been evaluated for other dysbiosis-associated disorders [29].

As for molecular mechanisms, it was demonstrated that both HSCs and Kupffer cells as well as hepatocytes were sensitive to LPS by expressing TLR4. An important finding of Dapito et al. [40] was that hepatocarcinogenesis in chronically injured liver depended on the gut microbiota and TLR4 activation in non-bone-marrow-derived resident liver cells, including both hepatocytes and HSCs. These results were different from those of Yu et al. [39], who found that Kupffer cells were the main targets of LPS/TLR4 signals in the liver and played a pivotal role in the induction of TNF $\alpha$ and IL-6. Inactivation of Kupffer cells has been shown to cause a significant reduction in cytokine production and complementary proliferation in response to DEN. Thus, further studies are required to distinguish the relative contribution of TLR4 expressed in different cell types to hepatocarcinogenesis. In addition, Dapito et al. [40] reported that the LPS/TLR4 pathway mediated the expression of the epiregulin hepatomitogen, which belongs to the epidermal growth factor family. Hepatomitogen will lead to activation of epidermal growth factor in the first stages of DEN/CCl 4 carcinogenesis and to reduction of hepatocyte apoptosis by NF- $\mathrm{\kappa B}$ nuclear translocation. Therefore, the therapeutic benefit of TLR4 in patients with high risk of HCC needs to be explored.

\section{Probiotics and HCC}

Gut microbiota are thought to contribute to the progression of HCC through the gut-liver axis [42]. Consequently, modulation of the gut microbiota by probiotics may represent a new avenue to treat or prevent the development of HCC. Alterations of the type and amount of gut microbiota not only promote gut flora balance, intestinal inflammation and mucosal barrier function, but also dramatically improve the cirrhotic condition and prevent the occurrence of HCC [19]. Interestingly, probiotics could inhibit the translocation of endotoxin, which bears PAMPs, and activate the damage-associated molecular patterns (DAMPs) such as highmobility group box 1 [19]. Also, probiotic strains are a safer and less expensive therapeutic approach.

Several methods, including the use of prebiotics, probiotics and synbiotics, could be applied to prevent overgrowth of gut microbiota [43]. They improved derangement in flora by decreasing the counts of pathogenic bacteria and thus improving the endotoxemia [44]. Ideal probiotic strains have special properties, such as stimulation of the immune system, thereby improving intestinal function via adhering and colonizing the intestinal epithelium. Furthermore, when these strains stimulate the immune system, they must be able to survive and can exert considerable healthful outcomes [45].

Probiotics are live microorganisms (according to the FAO/WHO); when administered in adequate amounts they will confer a healthful benefit on the host [45], such as improving the balance of gut microbiota and stimulating the metabolites of bacteria products [46]. Prebiotics are a kind of non-absorbent oligosaccharide substances, such as lactulose; in order to 
adjust the intestinal microflora balance, they can promote the growth of beneficial bacteria and inhibit the harmful ones [47]. Synbiotics refers to a complex of probiotics and prebiotics and are believed to be more efficient compared to either of the two alone [48].

The plasma endotoxin level increases in patients with HBV and HCV [44, 49]. Furthermore, high amounts of the proinflammatory cytokines described above in these patients cause liver damage in the longer run. Results from a study in patients with HBV and HCV infection showed that alleviation of endotoxemia could be achieved by increasing bifidobacteria and lactobacilli numbers and avoiding the growth of pathogens. However, regarding liver damage more studies are required to properly assess the benefits of probiotic therapy [50].

The colonic microbiota of cirrhosis patients are different from those of healthy control subjects [51]. Increases in Enterobacteriaceae and Enterococcus with a reduction in Bifidobacterium species were noted in one report [26]. Bacteriotherapy with probiotic strains in patients with cirrhosis can modulate the bioecological system via prevention of growth of pathogens, improvement to the mucosal layer, protection of intestinal epithelia cells and reduction of bacterial translocation. All of these mechanisms will decrease portal hypertension owned to the inhibition of nitrogen monoxide production followed by lowering plasma LPS. Changes in gut microbiota may also play a role in the pathogenesis of other complications of cirrhosis (e.g. spontaneous bacterial peritonitis, hepatorenal syndrome and hepatic encephalopathy [49].

Few studies were performed to assess probiotic effects on the toxicity of aflatoxin in liver dysfunction and HCC. In a research performed by El-Nezami et al. [52], diminution of aflatoxin concentration was observed in fecal samples after the administration of Lactobacillus rhamnosus LC705. Five weeks later, they collected the L. rhamnosus LC705 together with Propionibacterium freudenreichii subspecies. The ingestion of the probiotic capsule was confirmed by the concentration of L. rhamnosus LC705 in fecal samples. In the subjects who received the probiotic mixture, L. rhamnosus LC705 constituted the major part of the fecal Lactobacillus population, whereas in the group without probiotic mixture, the bacterium was absent. In this study the authors showed that a probiotic supplement reduced the biological dose of aflatoxin exposure and may offer an effective dietary approach to decrease the risk of HCC.

\section{Conclusions}

Growing evidence is showing that gut microbiota are associated with oncogenic pathways that increasingly promote the development of HCC. Modulation of the gut microflora by antibiotics and probiotics may represent novel strategies to prevent the progression from chronic hepatitis to liver cirrhosis and HCC. However, many questions remain unclear regarding the roles of the microbiota in the occurrence of HCC. Further studies need to be performed on how the microbiota are assembled as well as on which factors contribute to their long-term stability in both health and illness. It seems that the future roles of probiotics, prebiotics and synbiotics may go beyond traditional gastrointestinal illnesses.

\section{Disclosure Statement}

The authors declare that no conflict of interest exists. 


\section{References}

1 El-Serag HB: Hepatocellular carcinoma. N Engl J Med 2011;365:1118-1127.

2 Mandair DS, Rossi RE, Pericleous M, Whyand T, Caplin M: The impact of diet and nutrition in the prevention and progression of hepatocellular carcinoma. Expert Rev Gastroenterol Hepatol 2014;8:369-382.

3 Shah VJ, Kamath PS: Portal hypertension and gastrointestinal bleeding; in Feldman M, Friedman LS, Brandt LJ (eds): Sleisenger and Fordtran's Gastrointestinal and Liver Disease, ed 9. Philadelphia, Saunders Elsevier, 2010, pp 1489-1516.

4 Madsen BS, Havelund T, Krag A: Targeting the gut-liver axis in cirrhosis: antibiotics and non-selective $\beta$-blockers. Adv Ther 2013;30:659-670.

5 Ohtani N: Microbiome and cancer. Semin Immunopathol 2015;37:65-72.

6 Qin J, Li R, Raes J, Arumugam M, Burgdorf KS, Manichanh C, Nielsen T, Pons N, Levenez F, Yamada T, Mende DR, Li J, Xu J, Li S, Li D, Cao J, Wang B, Liang H, Zheng H, Xie Y, Tap J, Lepage P, Bertalan M, Batto JM, Hansen T, Le Paslier D, Linneberg A, Nielsen HB, Pelletier E, Renault P, Sicheritz-Ponten T, Turner K, Zhu H, Yu C, Li S, Jian M, Zhou Y, Li Y, Zhang X, Li S, Qin N, Yang H, Wang J, Brunak S, Doré J, Guarner F, Kristiansen K, Pedersen O, Parkhill J, Weissenbach J; MetaHIT Consortium, Bork P, Ehrlich SD, Wang J: A human gut microbial gene catalog established by metagenomic sequencing. Nature 2010;464:59-65.

7 Wiest R, Lawson M, Geuking M: The pathogenesis of gastrointestinal bacterial overgrowth. J Hepatol 2014;60: 197-209.

8 Douhara A, Moriya K, Yoshiji H, Noguchi R, Namisaki T, Kitade M, Kaji K, Aihara Y, Nishimura N, Takeda K, Okura Y, Kawaratani H, Fukui H: Reduction of endotoxin attenuates liver fibrosis through suppression of hepatic stellate cell activation and remission of intestinal permeability in a rat non-alcoholic steatohepatitis model. Mol Med Rep 2015;11:1693-1700.

9 Van Leeuwen PA, Boermeester MA, Houdijk AP, Ferwerda CC, Cuesta MA, Meyer S, Wesdorp RI: Clinical significance of translocation. Gut 1994;35(suppl 1):S28-S34.

10 Jirillo E, Caccavo D, Magrone T, Piccigallo E, Amati L, Lembo A, Kalis C, Gumenscheimer M: The role of the liver in the response to LPS: experimental and clinical findings. J Endotoxin Res 2002;8:319-327.

11 Han DW: Intestinal endotoxemia as a pathogenetic mechanism in liver failure. World J Gastroenterol 2002;8: 961-965.

12 Schuppan D, Afdhal NH: Liver cirrhosis. Lancet 2008;371:838-851.

13 Sandler NG, Koh C, Roque A, Eccleston JL, Siegel RB, Demino M, Kleiner DE, Deeks SG, Liang TJ, Heller T, Douek DC: Host response to translocated microbial products predicts outcomes of patients with HBV or HCV infection. Gastroenterology 2011;141:1220-1230, 1230.e1-3.

14 Li DY, Yang M, Edwards S, Ye SQ: Nonalcoholic fatty liver disease: for better or worse, blame the gut microbiota? JPEN J Parenter Enteral Nutr 2013;37:787-793.

15 Zare-Bidaki M, Tsukiyama-Kohara K, Arababadi MK: Toll-like receptor 4 and hepatitis B infection: molecular mechanisms and pathogenesis. Viral Immunol 2014;27:321-326.

16 Munteanu D, Negru A, Radulescu M, Mihailescu R, Arama SS, Arama V: Evaluation of bacterial translocation in patients with chronic HCV infection. Rom J Intern Med 2014;52:91-96.

17 Gómez-Hurtado I, Such J, Sanz Y, Francés R: Gut microbiota-related complications in cirrhosis. World J Gastroenterol 2014;20:15624-15631.

18 Cirera I, Bauer TM, Navasa M, Vila J, Grande L, Taurá P, Fuster J, García-Valdecasas JC, Lacy A, Suárez MJ, Rimola A, Rodés J: Bacterial translocation of enteric organisms in patients with cirrhosis. J Hepatol 2001;34:32-37.

19 Zhang HL, Yu LX, Yang W, Tang L, Lin Y, Wu H, Zhai B, Tan YX, Shan L, Liu Q, Chen HY, Dai RY, Qiu BJ, He YQ, Wang C, Zheng LY, Li YQ, Wu FQ, Li Z, Yan HX, Wang HY: Profound impact of gut homeostasis on chemicallyinduced pro-tumorigenic inflammation and hepatocarcinogenesis in rats. J Hepatol 2012;57:803-812.

20 Ilan Y: Leaky gut and the liver: a role for bacterial translocation in nonalcoholic steatohepatitis. World J Gastroenterol 2012;18:2609-2618.

21 Dhiman RK: Gut microbiota and hepatic encephalopathy. Metab Brain Dis 2013;28:321-326.

22 Bajaj JS: The role of microbiota in hepatic encephalopathy. Gut Microbes 2014;5:397-403.

23 Pijls KE, Jonkers DM, Elamin EE, Masclee AA, Koek GH: Intestinal epithelial barrier function in liver cirrhosis: an extensive review of the literature. Liver Int 2013;33:1457-1469.

24 Gou YZ, Lian JQ, Nie QH: Bacterial translocation and its consequences in patients with liver cirrhosis (in Chinese). Zhonghua Gan Zang Bing Za Zhi 2006;14:796-797.

25 Choi Y, Jeon WK, Hwang SJ, Kim BI, Sohn CI, Park DI, Cho YK, Kim HJ, Park JH: The role of the gut barrier function in the pathophysiology of viral liver cirrhosis. Hepatogastroenterology 2011;58:1244-1247.

26 Sharma V, Garg S, Aggarwal S: Probiotics and liver disease. Perm J 2013;17:62-67.

27 Seki E, De Minicis S, Osterreicher CH, Kluwe J, Osawa Y, Brenner DA, Schwabe RF: TLR4 enhances TGF-beta signaling and hepatic fibrosis. Nat Med 2007;13:1324-1332.

28 Fox JG, Feng Y, Theve EJ, Raczynski AR, Fiala JL, Doernte AL, Williams M, McFaline JL, Essigmann JM, Schauer DB, Tannenbaum SR, Dedon PC, Weinman SA, Lemon SM, Fry RC, Rogers AB: Gut microbes define liver cancer risk in mice exposed to chemical and viral transgenic hepatocarcinogens. Gut 2010;59:88-97.

29 Roderburg C, Luedde T: The role of the gut microbiome in the development and progression of liver cirrhosis and hepatocellular carcinoma. Gut Microbes 2014;5:441-445. 
30 Seki E, Schnabl B: Role of innate immunity and the microbiota in liver fibrosis: crosstalk between the liver and gut. J Physiol 2012;590:447-458.

31 Pagnini C, Saeed R, Bamias G, Arseneau KO, Pizarro TT, Cominelli F: Probiotics promote gut health through stimulation of epithelial innate immunity. Proc Natl Acad Sci USA 2010;107:454-459.

32 Fukata M, Chen A, Vamadevan AS, Cohen J, Breglio K, Krishnareddy S, Hsu D, Xu R, Harpaz N, Dannenberg AJ, Subbaramaiah K, Cooper HS, Itzkowitz SH, Abreu MT: Toll-like receptor-4 promotes the development of colitis-associated colorectal tumors. Gastroenterology 2007;133:1869-1881.

33 Karnati HK, Pasupuleti SR, Kandi R, Undi RB, Sahu I, Kannaki TR, Subbiah M, Gutti RK: TLR-4 signalling pathway: MyD88 independent pathway up-regulation in chicken breeds upon LPS treatment. Vet Res Commun 2015;39:73-78.

34 Dolganiuc A, Norkina O, Kodys K, Catalano D, Bakis G, Marshall C, Mandrekar P, Szabo G: Viral and host factors induce macrophage activation and loss of toll-like receptor tolerance in chronic HCV infection. Gastroenterology 2007;133:1627-1636.

35 Schwabe RF, Jobin C: The microbiome and cancer. Nat Rev Cancer 2013;13:800-812.

36 Ray K: Gut microbiota: obesity-induced microbial metabolite promotes HCC. Nat Rev Gastroenterol Hepatol 2013;10:442.

37 Yoshimoto S, Loo TM, Atarashi K, Kanda H, Sato S, Oyadomari S, Iwakura Y, Oshima K, Morita H, Hattori M, Honda K, Ishikawa Y, Hara E, Ohtani N: Obesity-induced gut microbial metabolite promotes liver cancer through senescence secretome. Nature 2013;499:97-101.

38 Kitazawa S, Denda A, Tsutsumi M, Tsujiuchi T, Hasegawa K, Tamura K, Maruyama H, Konishi Y: Enhanced preneoplastic liver lesion development under 'selection pressure' conditions after administration of deoxycholic or lithocholic acid in the initiation phase in rats. Carcinogenesis 1990;11:1323-1328.

39 Yu LX, Yan HX, Liu Q, Yang W, Wu HP, Dong W, Tang L, Lin Y, He YQ, Zou SS, Wang C, Zhang HL, Cao GW, Wu MC, Wang HY: Endotoxin accumulation prevents carcinogen-induced apoptosis and promotes liver tumorigenesis in rodents. Hepatology 2010;52:1322-1333.

40 Dapito DH, Mencin A, Gwak GY, Pradere JP, Jang MK, Mederacke I, Caviglia JM, Khiabanian H, Adeyemi A, Bataller R, Lefkowitch JH, Bower M, Friedman R, Sartor RB, Rabadan R, Schwabe RF: Promotion of hepatocellular carcinoma by the intestinal microbiota and TLR4. Cancer Cell 2012;21:504-516.

41 Toffanin S, Cornella H, Harrington A, Llovet JM: HCC is promoted by bacterial translocation and TLR-4 signaling: a new paradigm for chemoprevention and management. Hepatology 2012;56:1998-2000.

42 Chassaing B, Etienne-Mesmin L, Gewirtz AT: Microbiota-liver axis in hepatic disease. Hepatology 2014;59: 328-339.

43 Petschow B, Doré J, Hibberd P, Dinan T, Reid G, Blaser M, Cani PD, Degnan FH, Foster J, Gibson G, Hutton J, Klaenhammer TR, Ley R, Nieuwdorp M, Pot B, Relman D, Serazin A, Sanders ME: Probiotics, prebiotics, and the host microbiome: the science of translation. Ann NY Acad Sci 2013;1306:1-17.

44 Loguercio C, Federico A, Tuccillo C, Terracciano F, D’Auria MV, De Simone C, Del Vecchio Blanco C: Beneficial effects of a probiotic VSL\#3 on parameters of liver dysfunction in chronic liver diseases. J Clin Gastroenterol 2005;39:540-543.

45 Culligan EP, Hill C, Sleator RD: Probiotics and gastrointestinal disease: successes, problems and future prospects. Gut Pathog 2009;1:19.

46 Bengmark S: Bioecologic control of the gastrointestinal tract: the role of flora and supplemented probiotics and synbiotics. Gastroenterol Clin North Am 2005;34:413-436.

47 Fotiadis CI, Stoidis CN, Spyropoulos BG, Zografos ED: Role of probiotics, prebiotics and synbiotics in chemoprevention for colorectal cancer. World J Gastroenterol 2008;14:6453-6457.

48 Roberfroid M, Gibson GR, Hoyles L, McCartney AL, Rastall R, Rowland I, Wolvers D, Watzl B, Szajewska H, Stahl B, Guarner F, Respondek F, Whelan K, Coxam V, Davicco MJ, Léotoing L, Wittrant Y, Delzenne NM, Cani PD, Neyrinck AM, Meheust A: Prebiotic effects: metabolic and health benefits. Br J Nutr 2010;104(suppl 2):S1-S63.

49 Imani Fooladi AA, Mahmoodzadeh Hosseini H, Nourani MR, Khani S, Alavian SM: Probiotic as a novel treatment strategy against liver disease. Hepat Mon 2013;13:e7521.

50 Esposito E, Iacono A, Bianco G, Autore G, Cuzzocrea S, Vajro P, Canani RB, Calignano A, Raso GM, Meli R: Probiotics reduce the inflammatory response induced by a high-fat diet in the liver of young rats. J Nutr 2009;139: 905-911.

51 Bellot P, Francés R, Such J: Bacterial translocation in cirrhosis (in Spanish). Gastroenterol Hepatol 2008;31: 508-514.

52 El-Nezami HS, Polychronaki NN, Ma J, Zhu H, Ling W, Salminen EK, Juvonen RO, Salminen SJ, Poussa T, Mykkänen HM: Probiotic supplementation reduces a biomarker for increased risk of liver cancer in young men from Southern China. Am J Clin Nutr 2006;83:1199-1203. 